\title{
EXTERNAL OPTICAL MODULATOR USING A LOW-COST FABRY-PEROT LASER DIODE FOR OPTICAL ACCESS NETWORKS
}

\author{
H. J. Lee ${ }^{1}$, H. Yoo ${ }^{2}$, Y. D. Jeong ${ }^{2}$, and Y. H. Won ${ }^{2}$ \\ ${ }^{1}$ Division of Information \& Communication Engineering, Kyungnam University, 449 \\ Wolyoung-dong, Masan,631-701, KOREA hyuek@kyungnam.ac.kr \\ ${ }^{2}$ Optical Internet Research Center (OIRC), Information and Communications University \\ (ICU),P.O.BOX77, Yusong-Gu, Daejeon,305-600,KOREA yhwon@icuac.kr
}

\begin{abstract}
We propose and demonstrate an external optical modulation method based on TE/TM-mode absorption nulls in a Multiple Quantum Well (MQW) Fabry-Perot laser diode (FP-LD). The center wavelength of the absorption nulls is rapidly shifted to short-wavelength by the small current change $(\sim$ $1 \mathrm{~mA}$ ) in the FP-LD, which can modulate optical signal with more than $10 \mathrm{~dB}$ of extinction ratio (ER). The shift of the center wavelength comes from the refractive index change due to anomalous dispersion and plasma effect in MQW FP-LD waveguide. Non-inverting and inverting signals are made by TE- and TM-mode absorption nulls at 155.52 Mbps and BERs for the signals are measured, respectively.
\end{abstract}

\section{INTRODUCTION}

Recently, Wavelength Division Multiplexing (WDM)-Passive Optical Network (PON) for optical access networks has received a great deal of an attention. WDM-PON has a high security, a protocol transparency, and wide bandwidth compared with Time Division Multiplexing (TDM)-PON. However, WDM-PON typically requires high-cost DFB-LDs, which becomes a big bottleneck for a commercial deployment. Therefore, many researchers have been intensively trying to find out cost-effective methods for WDM-PON [1-3]. One of the methods is a spectrum-sliced WDM source by optically filtering of an incoherent broadband Amplified Spontaneous Emission (ASE) light such as a LED 
or an EDFA [1]. The others are based on injection-locked Fabry-Perot laser diode (FP-LD) sources by external coherent [2] or incoherent [3] light. However, the injection-locked FP-LD source may have a demerit of potentially unstable operation by unexpected behaviours inside the laser diode [4]. On the other hand, the LED light source may be a good candidate for WDM-PON, but its output power is not enough for the purpose. In case of the EDFA, the spectrum-sliced source has relatively much higher output power than the LED, but expensive external optical modulators [1] or SOAs [5] for signal modulation are needed.

In this paper, we propose and demonstrate an external optical modulator employing a low-cost FP-LD, which can be effectively used for WDM-PON as an optical access network application. The modulation is based on the shift of TE/TM-mode absorption nulls (by refractive index change) due to anomalous dispersion and plasma effect in a FP-LD. The optical modulated signal with high extinction ratio $(>10 \mathrm{~dB}$ ) at $155.52 \mathrm{Mbps}$ can be achieved. Based on the proposed optical modulator, a cost-effective WDM-PON architecture is proposed.

\section{PRINCIPLE}

When a multiple-quantum-well (MQW) type FP-LD is driven over a threshold current, the FP-LD makes light by lasing on TE-mode. On the other hand, TMmode in the FP-LD shows only absorption nulls not lasing owing to very small TM-gain inside the Fabry-Perot mirror (resonator). If no current is applied to the FP-LD, both modes (TE- and TM-mode) have absorption nulls. As the current increases from $0 \mathrm{~mA}$ to the threshold current, all the absorption nulls are moved to short-wavelength without any lasing on both modes. Even though light due to spontaneous emission occurs, the light at small current level can be ignored in comparison to externally incident light for optical modulation. The wavelength of the incident light is spectrally aligned with the center wavelength of one among the absorption nulls. Thus, optical modulation can be achieved by vibrating the center wavelength of the null due to current signal injection into a PF-LD.

The shift of the absorption null comes from the change of the refractive index in an MQW FP-LD waveguide, which is due to anomalous dispersion, plasma effect, and bandgap shrinkage [6]. However, the bandgap shrinkage can be neglected when an input light wavelength is longer than a bandgap wavelength of the waveguide in the MQW FP-LD [7]. In this case, the anomalous dispersion and the plasma effect become dominant for the refractive index change. The refractive index change due to the plasma effect in a bulky waveguide can be written as follow $[6,8]$. 


$$
\Delta n=\frac{e^{2} N}{2 \omega^{2} \varepsilon_{0} \varepsilon_{r} m_{c}^{*}} n
$$

where, $N$ is a carrier density, $n$ is the refractive index in the absence of the plasma effect, $\varepsilon_{0} \varepsilon_{\mathrm{r}}$ is the dielectric constant of the active region, and $m_{c}^{*}$ is effective mass of the carrier. The total refractive index change due to plasma effect in a MQW waveguide can be easily obtained by summing refractive index changes in wells and barriers [6]. In (1), because $\omega=2 \pi / \lambda$, the $\sqrt{\Delta n}$ is proportional to an input light wavelength $\lambda$. On the contrary, a refractive index change due to the anomalous dispersion according to the wavelength $\lambda$ is reduced. Therefore, as the input light wavelength increases, the plasma effect is more dominant than the anomalous dispersion. As a result, the total refractive index change becomes almost constant for a wide range of wavelength $(>100 \mathrm{~nm})[6]$.

Let us consider a modulation speed for the proposed PF-LD modulator. To operate a PF-LD as an optical modulator, the injection current $J$ with less than a threshold current $J_{t h}$ must be applied, i.e. carrier density $N<$ threshold density $N_{t h}$. As a consequence, a photon density $N_{p h}$ becomes almost zero, which means a stimulated emission can be neglected. Thus, the rate equation for the carrier density $N$ can be expressed as [9]

$$
\frac{d N}{d t}=\frac{J}{q d}-\frac{N}{\tau_{e}(N)}
$$

where, $q$ and $d$ are the charge constant and the thickness of the active layer, respectively. And, $1 / \tau_{e}(N)$ is the effective recombination rate, which $1 / \tau_{e}(N)=A+B N+C N^{2}$, where $A N$ is the nonradiative recombination rate, $B N^{2}$ is the radiative spontaneous rate, and $C N^{3}$ is the Auger recombination rate. Because of the carrier dependence term $\tau_{e}(N)$, Eq. (2) is nonlinear and can be solved numerically or approximately. Assuming $\tau_{e}$ is constant, i.e. $B=C=0$, we can know easily that the carrier density $N(t)$ increases logarithmically and decays exponentially with the time constant $\tau_{e}$. Thus, the speed for the proposed modulator strongly depends on $\tau_{e}$. By the calculation from typical parameter values $\left(\mathrm{A}=5 \times 10^{8}, \mathrm{~B}=1 \times 10^{-10}, \mathrm{C}=3 \times 10^{-29}, N_{0}=1 \times 10^{18}\right.$, and $\left.N_{t h}=2.61 \times 10^{18}\right)$ in [9], $\tau_{e}\left(N_{0}\right) \approx 1.58 \mathrm{nsec}$ and $\tau_{e}\left(N_{t h}\right) \approx 1.04 \mathrm{nsec}$, which mean roughly $630 \mathrm{Mbps} \sim 1$ Gbps modulation speed.

\section{EXPERIMENTAL RESULTS}

Fig. 1 shows the experimental result for absorption nulls with respect to the injection current in a FP-LD, which the laser diode used in the experiment is 
InGaAsP MQW type. The absorption nulls are made by being kept and dissipated in a FP-LD cavity for the input light with the wavelength that the phase after each round trip has to be an integral multiple of $2 \pi$. In other words, $\lambda_{p}=2 n L / p$, where $\lambda_{p}$ is the wavelength of the $p$ th cavity mode, $L$ is the cavity length. When a current is injected, all the absorption nulls are continuously shifted to shortwavelength by negative refractive index change. But, for over the threshold current $\left(\mathrm{I}_{\mathrm{TH}}=\sim 11 \mathrm{~mA}\right.$ ), the nulls start to move in the opposite direction (to longwavelength) due to the thermal effect by lasing [10]. Here, it is worthy noting that the nulls are rapidly shifted to short-wavelength by a small amount of current from 0 to $1 \mathrm{~mA}$ as shown in Fig. 2. The center wavelength of the nulls is simultaneously moved by $\sim 0.25 \mathrm{~nm}(\sim 30 \mathrm{GHz})$ due to only $1 \mathrm{~mA}$ current, which is enough to modulate optical signal. The absorption null has the notch filter characteristic of $\sim 0.1 \mathrm{~nm}$ bandwidth at $-3 \mathrm{~dB}$ and $\sim 19 \mathrm{~dB}$ attenuation at the center wavelength. For the small current level such as $1 \mathrm{~mA}$, the spontaneous emission noise generated by the FP-LD itself is negligible compared to the input light.

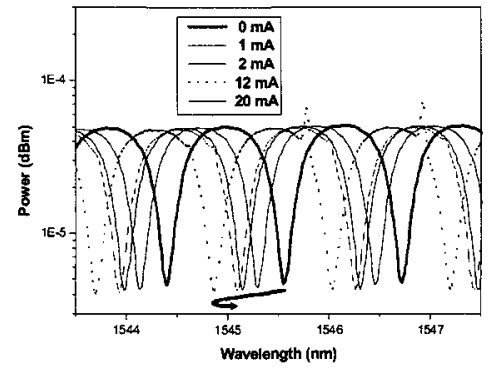

Figure 1 TM-mode absorption spectra of Fabry-Perot laser-diode (FP-LD) with the current change from 0 to $20 \mathrm{~mA}$.

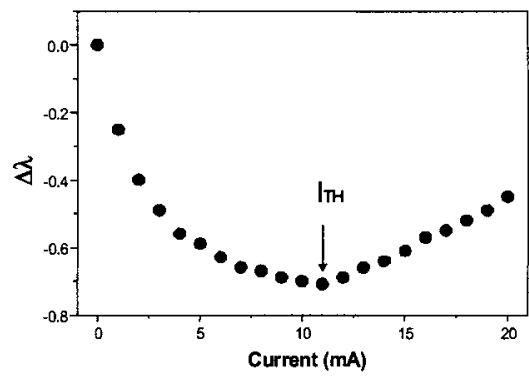

Figure 2 Center wavelength displacement of the absorption null versus injection current change.

Fig. 3 shows the experimental setup for an external optical modulation using a low-cost FP-LD. Laser light from the tunable-LD is incident into the FP-LD through PC (Polarization controller)1, OC (Optical Circulator), PBS (Polarization Beam Splitter) and $\mathrm{PC} 2$. By the $\mathrm{PC}$, the polarization (TE or TM) of the incident light into the FP-LD can be determined. The reflected light from the FP-LD comes out of 3 port of the OC through the PBS. The output signal may have an inverted or a non-inverted data format, which depends on which side (short-wavelength side $\rightarrow$ inverting, long-wavelength side $\rightarrow>$ non-inverting) of absorption nulls is used. BERs for the inverted and the non-inverted signal on TE- and TM-mode absorption null at 155.52 Mbps ( $2^{31}-1$ PRBS) are measured and shown in Fig. 4, respectively. Non-inverted signal that is made on long-wavelength side of the TM-mode absorption null, shows the best BER performance. The worst case, i.e. inverted data signal on the short-wavelength side of the TE-mode absorption null, has $\sim 2.3$ 
dB power penalty $\left(@ 10^{-9}\right)$. The minimum and the maximum insertion loss of the proposed modulator are $10.2 \mathrm{~dB}$ and $13.5 \mathrm{~dB}$ for the non-inverted signal on the TM-mode null and for the inverted signal on the TE-mode null, respectively. ERs for all the data show more than $10 \mathrm{~dB}$.

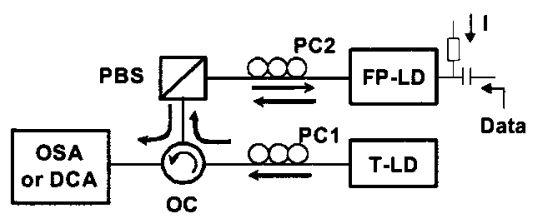

Figure 3 The experimental setup for the proposed external modulator based on the absorption null modulation of a FP-LD.

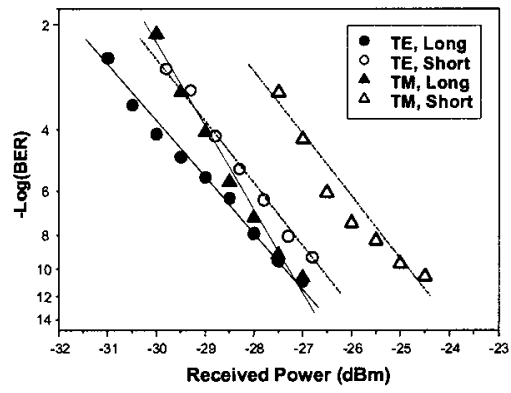

Figure 4 Measured BER curves and the corresponding eye diagrams for $(\mathbf{O})$ non-inverted, $(\mathrm{O})$ inverted data signal by a TE-mode null and $(\boldsymbol{A})$ non-inverted, $(\triangle)$ inverted data signal by a TM-mode null.

The MQW FP-LD used in the experiment has a cavity length of $\sim 300$ um and then shows that the wavelength difference between nulls as shown in Fig. 1 is around $1.17 \mathrm{~nm}$. For the WDM signal with the ITU-T grid, the FP-LD has to be designed to get the optimal cavity length. In order to know the wavelength sensitivity for the proposed FP-LD modulator, we measured ERs and insertion losses from $1520 \mathrm{~nm}$ to $1630 \mathrm{~nm}$ at $155.52 \mathrm{Mbps}$ for non-inverting signal on TMmode nulls as shown in Fig. 5. The proposed FP-LD modulator shows good performances (ERs $>11.5 \mathrm{~dB}$ and insertion losses $<-13.4 \mathrm{~dB}$ ) for very wide wavelength range $(\sim 100 \mathrm{~nm})$. This result is in very good agreement with the result in [6].

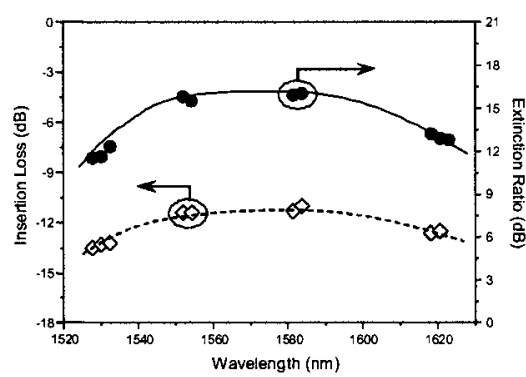

Figure 5 Measured Extinction Ratio (dB) and Insertion Loss (dB) corresponding to Wavelength.

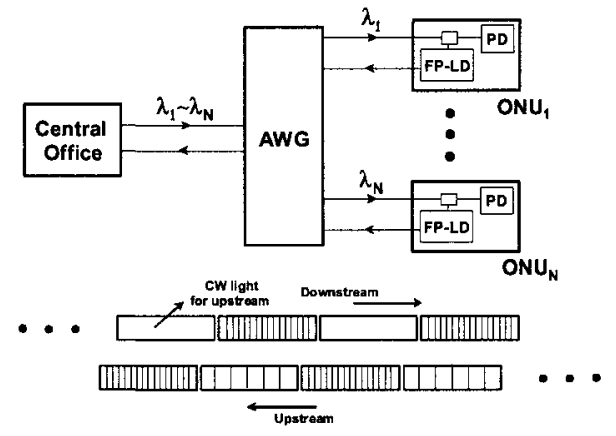

Figure 6 WDM-PON architecture with the proposed FP-LD modulator. 


\section{APPLICATION TO WDM-PON}

Fig. 6 shows one example of WDM-PON based on the proposed method. Instead of a DFB-LD source (employed in typical WDM-PON) inside the optical network unit (ONU), the proposed FP-LD modulator is used. WDM downstream signals with wavelength $\lambda_{1} \sim \lambda_{\mathrm{N}}$ from the central office are wavelength-routed and transmitted to ONUs. As shown in Fig. 6, each signal from the central office is composed of the modulated downstream data for half and the $\mathrm{CW}$ light for the other half. Here, the $\mathrm{CW}$ light is used for the generation of upstream signal by the FP-LD modulator at the ONU and then routed back to the central office. Similar architectures had been proposed [2,11]. But, in [2], very high-speed downstream signal is required to make the single mode operation of a FP-LD by injectionlocking, which may be potentially unstable. Also, in [11], high-cost optical modulators are needed. On the contrary, the proposed modulator is based on the absorption nulls in a PF-LD and thus shows very stable operation in a costeffective way.

\section{CONCLUSIONS}

In this paper, new optical modulation method using a low-cost FP-LD has proposed and experimentally demonstrated. Due to the cost-effectiveness, the proposed optical modulator has many applications in optical access networks, optical signal processing, and so on. Even though the modulation speed in the experiment is $155.52 \mathrm{Mbps}$ with more than $10 \mathrm{~dB}$ insertion loss, $\sim 1 \mathrm{Gbps}$ modulation and low $(\sim 6 \mathrm{~dB})$ insertion loss will be possible with the small modification of a FP-LD such as a length and a reflectance control for the FP cavity.

\section{ACKNOWLEDGMENTS}

This work was supported by the Korean Science and Engineering Foundation (KOSEF) through OIRC project in ICU. 


\section{REFERENCES}

[1] J.S. Lee, Y.C. Chung and D.J. DiGiovanni, "Spectrum-sliced fiber amplifier light source for multichannel WDM applications," IEEE Photon. Technol. Lett., vol. 5, pp. 1458$1461,1993$.

[2] L.Y. Chan, C.K. Chan, D.T.K. Tong, F. Tong and L.K. Chen, "Upstream traffic transmitter using injection-locked Fabry-Perot laser diode as modulator for WDM access networks," Electron. Lett., vol. 38, pp. 43-45, 2002.

[3] H.D. Kim, S.G. Kang and C.H. Lee, "A low-cost WDM source with an ASE injected Fabry-Perot semiconductor laser," IEEE Photon. Technol. Lett., vol. 12, pp. 1067-1069, 2000.

[4] G. Yabre, H. Waardt, H.P.A. Boom and G.-D. Khoe, "Noise characteristics of singlemode semiconductor lasers under external light injection," IEEE J. Quantum Electron., vol. 36, pp. 385-393, 2000.

[5] P. Healey, P. Townsend, C. Ford, L. Johnston, P. Townley, I. Lealman, L. Rivers, S. Perrin and R. Moore, "Spectral slicing WDM-PON using wavelength-seeded reflective SOAs," Electron. Lett., vol. 37, pp. 1181-1182, 2001.

[6] J.I. Shim, M. Yamaguchi and M. Kitamura, "Refractive index and loss changes produced by current injection in InGaAs(p)-InGaAsP Multiple Quantum-Well (MQW) waveguides," IEEE J. Select. Topics Quantum Electron., vol. 1, pp. 408-415, 1995.

[7] B.R. Bennett, R.A. Soref and J.A. Del Alamo, "Carrier-induced change in refractive index of InP, GaAs, and InGaAsP," IEEE J. Quantum Electron., vol 29, pp. 113-122, 1990.

[8] K. Iizuka, Elements of Photonics, Volume II, Chapter 14, Wiley \& Sons, New York, N.Y., 2002.

[9] M.M.-K., Liu, Principles and applications of optical communications, Chapter 12, IRWIN, Times Mirror Higher Education Group, Inc., 1996.

[10] T. Higashi, T. Yamamoto, S. Ogita and M. Kobayashi, "Experimental analysis of temperature dependence of oscillation wavelength in quantum-well FP semiconductor lasers," IEEE J. Quantum Electron., vol. 34, pp. 1680-1689, 1998.

[11] N.J. Frigo, P.P. Iannone, P.D. Magill, T.E. Darcie, M.M. Downs, B.N. Desai, U. Koren, T.L. Kock, C. Dragone, H.M. Presby and G.E. Bodeep, "A wavelength-division multiplexed passive optical network with cost-shared components," IEEE Photon. Technol. Lett., vol. 6, pp. 1365-1367, 1994. 